\title{
La accesibilidad en los videojuegos: una asignatura pendiente
}

\author{
Accessibility in the video games is \\ a pending matter
}

\section{Palabras clave}

Accesibilidad, tecnologías de la información, videojuegos, Boccia, Slalom.

\section{Keywords}

Accessibility, information technology, videogames, Boccia, Slalom.
En los últimos años, el avance de las tecnologías de la información (TIC) ha supuesto un profundo cambio en diferentes ámbitos de nuestra vida, tanto que a día de hoy resulta complicado imaginar cómo sería ésta sin ordenadores o teléfonos móviles.

En 1952 Alexander S. Douglas, un estudiante de la universidad de Cambridge, programó el primer videojuego de la historia, llamado OXO (Winter, 2010). Este juego digital era una versión gráfica del tres en raya, que aunque hoy por hoy sea uno de los juegos más conocidos, en su momento no llegó a alcanzar el éxito esperado. Sin embargo, en los últimos años los videojuegos han ido evolucionando hasta desempeñar un papel preponderante en nuestra sociedad (Flynn et al., 2007: I80).

En España desde el año 2009, la accesibilidad ha experimentado un gran auge. A pesar de ello, existe la necesidad de mejorar la accesibilidad de los videojuegos (Baranowski et al., 2008: 74) para todo tipo de usuarios, independientemente sea cual sea su grado de capacidad, promoviendo así una sociedad más inclusiva y garantizando el derecho al acceso universal e igualitario a la cultura y al ocio (ONU, 2006).
Ma Isabel Díez Alegre

<idiezalegre@hotmail.com>

Centro de Referencia Estatal para la Atención a Personas con Grave Discapacidad y para la Promoción de la Autonomía Personal y Atención a la Dependencia, San Andrés del Rabanedo, IMSERSO

Para citar:

Díez Alegre, M.I. (20I3): “La accesibilidad en los videojuegos: una asignatura pendiente". Revista Española de Discapacidad, I (2): I $55^{-I} 58$.

<http://dx.doi.org/IO.5569/23405IO4.OI.O2.IO> 
Según International Game Developers Association (IGDA), definimos accesibilidad en videojuegos (IGDA, 2004) como la habilidad de jugar a un juego bajo condiciones restrictivas ya sea por una limitación funcional o por una discapacidad. De esta forma, se permite la interacción del usuario con el medio eliminando las barreras de accesibilidad.

Actualmente, la mayoría de los videojuegos que incluyen accesibilidad están dirigidos a un colectivo específico (Abenójar, 20I2), como los juegos de un sólo botón (one switch games) indicado para participantes con movilidad reducida o juegos de audio destinados para jugadores con discapacidad visual. En contraposición, los autores (Savidis y Grammenos, 2006) apuestan por la accesibilidad universal de los videojuegos adaptados a cualquier tipo de jugador independiente de su discapacidad y no por videojuegos destinados a discapacidades específicas.

El empleo de los videojuegos aplicados al ámbito de la discapacidad cuenta con un gran valor potencial como recurso educativo y además es una herramienta tanto rehabilitadora como de ocio. En este sentido, al ser un medio interactivo precisa del papel activo del jugador de su implicación y motivación (Montgomery y Connolly, 2003). Según los últimos estudios sugieren que el uso de videojuegos puede ofrecer beneficios en la mejora de la coordinación visual y manual (Brown et al., 20I0: 575), la organización espacio-temporal, estimula la memoria (Cain et al., 20I 2: 64I), aumenta la autoestima (Díez y Cano, 20I 2: 23), así como favorece el contacto social (Hurkmans et al., 20IO: I 577) cuando se juega en grupo.

Por otra parte, el Centro de Referencia Estatal de Discapacidad y Dependencia (CRE) perteneciente al Imserso ha puesto a disposición dos videojuegos accesibles y gratuitos con menús sencillos, plantillas de alto contraste, locuciones o texto descriptivo en subtítulos. Ambos proyectos han sido testados por los usuarios del centro y todas las mejoras que se han ido aplicando al videojuego son fruto de la experiencia de los usuarios.
El primer videojuego "Boccia Virtual” es un proyecto pionero en Europa y ha sido desarrollado por la empresa asturiana Prometeo Innovations. Está basado en la Boccia, deporte paralímpico con gran desarrollo y reglamento internacional, similar a la petanca. Es considerado un juego de precisión y de estrategia. Son requisitos necesarios para poder jugar un ordenador y el mando de la videoconsola Nintendo Wii, que transmite los movimientos por medio de Bluetooth al ordenador. Aunque también es posible sustituir el mando por el botón izquierdo o derecho del ratón. Además existe la posibilidad de acoplar pulsadores a un ratón adaptado, y también incorpora el sistema de barrido y puede canalizar las órdenes a través de comandos de voz.

Por otra parte, con este videojuego se llevó a cabo un estudio (Díez y Cano, 20I2: 23) desde el Centro de Referencia Estatal de Discapacidad y Dependencia en colaboración con la Universidad Rey Juan Carlos de Madrid, donde participaron diez usuarios adultos con parálisis cerebral. El objetivo del estudio fue determinar si el entrenamiento de Boccia-Wii®, producía mejorías tanto a nivel físico como a nivel psicológico. Tras un periodo de dos meses y medio, con una hora y media de entrenamiento semanal, y con un número total de 30 sesiones se obtuvieron mejorías estadísticamente significativas en aspectos motores como la coordinación y la motricidad fina de la mano, el rango de movilidad activa, así como en la actividad muscular. A nivel psicológico se obtuvieron resultados positivos en la autoestima y la calidad de vida relacionada con la salud. Esta línea de investigación ha generado buenas prácticas mejorando tanto la calidad de la atención como la calidad de vida de los usuarios.

Por otra parte, el segundo videojuego es «Slalom. The Videogame» desarrollado por la empresa sevillana AccesAble-Games-The Game Kitchen. El Slalom es una modalidad deportiva que consiste en realizar un circuito, sorteando obstáculos en el menor tiempo posible. Al ser un videojuego accesible ofrece un gran abanico de posibilidades de configuración y un interfaz amigable con el fin de adaptarse a las necesidades 
de cada jugador. Entre las especificaciones accesibles nos permite configurar la velocidad del juego, el modo del control del joystick, el teclado o ratón, la configuración de la sensibilidad de los controles, el subtitulado de los sonidos, la elección del brillo y contraste de colores, entre otras. Dispone de varios tutoriales explicativos de las reglas para las diferentes pruebas. El objetivo sería adquirir habilidades mediante el juego en el manejo de la silla de ruedas y así poder transferir este conocimiento a su vida diaria. De esta forma esta herramienta interactiva ayudaría a mejorar su autonomía personal y su calidad de vida.

Estos dos videojuegos son ejemplo de accesibilidad. Ambos se pueden descargar gratuitamente desde la web del Centro de Referencia Estatal de Discapacidad y Dependencia (<www. crediscapacidadydependencia.es $>$ ). Importante destacar tres nuevas guías (de Abenójar et al., Barlet y Spohn, y Barnett y Humphreys, respectivamente) muy interesantes sobre la accesibilidad en videojuegos. A pesar de estos ejemplos, aún hay un largo camino por recorrer para que los videojuegos sean más inclusivos y ofrezcan mayor atención integral y de calidad en el ámbito de la discapacidad. Es necesario garantizar la equiparación de oportunidades, diseñando productos para todos, lo que implica responder a todo tipo de necesidades, exigiendo condiciones de accesibilidad. Por ello sería necesario impulsar más proyectos de Investigación Científica y Desarrollo Tecnológico desarrollando videojuegos accesibles empleados como recurso educativo o como una herramienta rehabilitadora y de ocio, incidiendo así en una mejora de la calidad de vida.

Referencias bibliográficas

Abenójar, V. et al. (2012): Buenas prácticas de accesibilidad en videojuegos. Madrid: Imserso.

Baranowski, T. et al. (2008); "Playing for real: video games and stories for health-related behavior change". American Journal of Preventive Medicine 34(I): 74-82.

Barlet, M.C. y Spohn, S.D. (2OI2): A practical guide to game accessibilty (en línea). <http://www. includification.com>, acceso I 5 de octubre 20 I 2.

Barnett, P. y Humphreys, S. (201 2): Game accessibility guidelines (en línea). <http://www. gameaccessibilityguidelines.com $>$, acceso I 5 de octubre 2012.

Brown, S.H. et al. (2010). "The effects of internetbased home training upper limb function in adults with cerebral palsy". Neurorehabil Neural Repair 24: 575-83.

Cain, M.S. et al. (2012): “Action video game experience reduces the cost of switching tasks".
Attention, Perception, \& Psychophysics 74(4): 64I-7.

Díez, M.I. y Cano, R. (20I2): "Empleo de un video juego como herramienta terapéutica en adultos con parálisis cerebral tipo tetraparesia espástica”. Estudio piloto. Fisioterapia. 34(I): 23-30.

Flynn, S. et al. (2007): "Feasibility of using the Sony Playstation 2 gaming platform for an individual poststroke: a case report". Journal of Neurologic Physical Therapy 3 I(4): I 80-9.

Hurkmans, H.L. et al. (2010): "Energy Expenditure in adults with cerebral palsy playing wii sports". Archives of Physical Medicine and Rehabilitation 9I(IO): I 577-8I.

International Game Developers Association. (2004): Accesibility in games: motivations and approaches (en línea). <http://archives.igda.org/ accessibility/IGDA_Accessibility_WhitePaper. pdf>, acceso I de noviembre de $20 \mathrm{I} 2$. 
Montgomery, P. y Connolly, B. (2003): Clinical Applications for motor control. Slack Incorporated.

Naciones Unidas (ONU) (2006): Convención internacional amplia e integral para la protección y promoción de los derechos y la dignidad de las personas con discapacidad (en línea). <http:// www.observatoriodeladiscapacidad.es/sites/ default/files/io/o2_capitulo2.pdf $>$, acceso 2I de octubre 2012.
Savidis, A. y Grammenos, D. (2006): Unified Design of universally accessible games (say what?) (en línea). <http://www.gamasutra. com/view/feature/I30I8I/unified_design_ of_universally_.php?page =I $>$, acceso $23 \mathrm{de}$ octubre de $20 \mathrm{I} 2$.

Winter, D. (2010): Noughts and crosses. The oldest graphical computer game (en línea). <http:// www.pong-story.com/I952.htm>, acceso I 8 de octubre $20 \mathrm{I} 2$. 